\title{
Influence of velocity profile on calibration function of Lorentz force flowmeter*
}

\author{
C. STELIAN ${ }^{1,2}$ ， Yang YU (于 洋) ${ }^{1,3}$, Ben-wen LI (李本文) ${ }^{3}, \quad$ A. THESS ${ }^{1,4}$ \\ (1. Institute of Thermodynamics and Fluid Mechanics, Ilmenau University of Technology, \\ Ilmenau 98684, Germany; \\ 2. Department of Physics, West University of Timisoara, \\ Timisoara 300233, Romania;
}

3. Key Laboratory of Electromagnetic Processing of Materials, Northeastern University,

Shenyang 110819, P. R. China;

4. Institute of Engineering Thermodynamics, German Aerospace Center,

Stuttgart 70569, Germany)

\begin{abstract}
A Lorentz force flowmeter is a noncontact electromagnetic flow-measuring device based on exposing a flowing electrically conducting liquid to a magnetic field and measuring the force acting on the magnet system. The measured Lorentz force is proportional to the flow rate via a calibration coefficient which depends on the velocity distribution and magnetic field in liquid. In this paper, the influence of different velocity profiles on the calibration coefficient is investigated by using numerical simulations. The Lorentz forces are computed for laminar flows in closed and open rectangular channels, and the results are compared with the simplified case of a solid conductor moving at a constant velocity. The numerical computations demonstrate that calibration coefficients for solid bodies are always higher than for liquid metals. Moreover, it can be found that for some parameters the solid-body calibration coefficient is almost twice as high as for a liquid metal. These differences are explained by analyzing the patterns of the induced eddy currents and the spatial distributions of the Lorentz force density. The result provides a first step for evaluating the influence of the laminar velocity profiles on the calibration function of a Lorentz force flowmeter.
\end{abstract}

Key words laminar flow, numerical simulation, electromagnetic velocimetry

Chinese Library Classification O361

2010 Mathematics Subject Classification 65Z05, 76D05, 76W05

\section{Introduction}

When a liquid metal flows in a pipe or in an open channel under the influence of the nonuniform magnetic field created by a permanent magnet, eddy currents are induced inside the liquid. These eddy currents create a Lorentz force opposite to the direction of the mean flow ${ }^{[1-2]}$. Moreover, the eddy currents induce an additional magnetic field which interacts with the permanent magnet in such a way as to create a force upon the magnet. By measuring these

* Received Sept. 26, 2013 / Revised Dec. 23, 2013

Project supported by the German Research Foundation (Deutsche Forschungsgemeinschaft)

Corresponding author C. STELIAN, Ph. D., E-mail: carmen_stelian@yahoo.com 
forces one can determine the velocity of the liquid metal. This noncontact electromagnetic flow measurement technique is called the Lorentz force velocimetry and permits flow measurement in hot and aggressive fluids such as liquid aluminum or molten stee ${ }^{[1-6]}$. When designing the flowmeters based on Lorentz force velocimetry, which is often called the Lorentz force flowmeters (LFF), it is important to know how strongly the measured force depends on the velocity profile in order to design the magnet systems in such a way as to minimize this profile-dependence. Whereas this question is reasonably well understood for laminar axi-symmetric pipe flows ${ }^{[4]}$, our understanding of this problem for open-channel flows with a free surface is not sufficiently advanced. The goal of the present work is to bridge this gap.

The specific motivation of the present work is illustrated in Fig. 1. The flow direction is perpendicular to the paper. Bold arrows indicate the magnetic field lines. The Lorentz force acts on both magnets and is in the same direction as the mean flow. The objective of the present work is to compute this Lorentz force for different flow rates and heights of the free surface. Figure 1 shows a highly simplified representation of the Lorentz force flowmeter used by Kolesnikov et al..$^{[5]}$ to determine the flow of liquid aluminium in secondary aluminium production. The flowmeter consists of two permanent magnets held together by a yoke which is connected to a force measurement system (not shown). The force measurement system records the Lorentz force which depends on the unknown volumetric flow rate, on the electrical conductivity of the liquid metal and on the spatial distribution of the magnetic field. Here, we are interested in the question how strongly the measured Lorentz force depends on the details of the velocity profile for a given flow rate and for a given liquid metal level. This question is of considerable practical importance because Lorentz force flowmeters are often calibrated by replacing the flowing liquid metal with a moving solid metal bar that has the same crosssection as the liquid metal. It is obvious that in this procedure, which is referred to as dry calibration $^{[7]}$, the velocity profile of a moving solid body is different from that in a liquid metal. It would therefore be important to know how much a calibration constant (to be defined below) determined from dry calibration differs from the desired "true" calibration constant for a real flow.

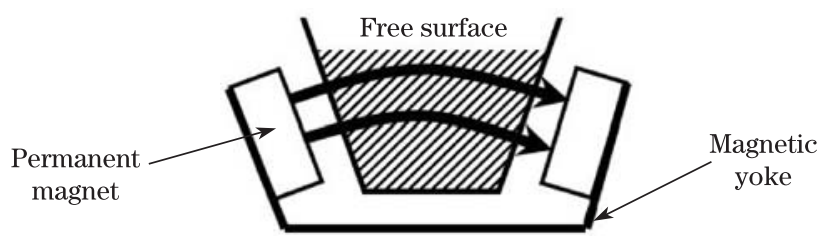

Fig. 1 Schematic of liquid metal flowing in open channel

To answer this question comprehensively, it would be necessary to numerically solve the full three-dimensional set of the Navier-Stokes equations and the Maxwell equations for the turbulent free-surface flow. Such a procedure is quite expensive computationally and does not provide insight into the general dependence of the Lorentz force on the key parameters of our problem ${ }^{[8]}$. We therefore define in Section 2 a highly simplified problem that is amenable to comparatively inexpensive numerical simulation and can be used to explore the Lorentz force for a wide range of geometry parameters. In Section 3, we present results of computations for the particular case when the two magnets are very small and can be represented by magnetic dipoles. Section 4 is devoted to the more general case of finite-size magnets. In Section 5, we summarize our conclusion and discuss topics that would be useful to investigate in future.

\section{Formulation of problem}

We simplify the situation shown in Fig. 1 to the greatest possible extent and consider the model shown in Fig. 2. A liquid or solid metal with width $\ell$ and height $h$ moves at mean velocity 
$v_{0}$ in the $z$-direction. The metal is exposed to two symmetrically placed permanent magnets whose magnetization is along the $x$-axis.

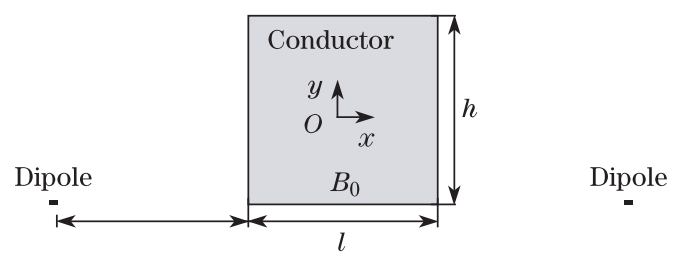

Fig. 2 Transversal cross section of simulated configuration with solid conductor and two dipoles

The movement of a fluid of electrical conductivity $\sigma$ in a magnetic field of induction $B$ induces electrical currents of density $j$, given as follows:

$$
j=\sigma(-\nabla \phi+v \times B) .
$$

where $\phi$ is the electric potential.

The interaction between eddy currents and the applied magnetic field is described by the Lorentz forces of density as follows:

$$
f=j \times B,
$$

The integrated Lorentz force over the metal volume, which is given by

$$
F=\int \sigma v(r) B(r)^{2} \mathrm{~d} V
$$

depends on both the magnetic field and the velocity distribution in the fluid. For this reason, it is very difficult to find an analytical expression which relates the Lorentz force to the mean velocity of the flow $v_{0}$. A simplified expression is given as follows:

$$
F=\sigma C_{v} v_{0},
$$

where the calibration coefficient depends on the magnetic field and velocity distribution $C_{v}(v, B)$. The dependence $F\left(v_{0}\right)$ in $(4)$ is linear in the case of laminar or turbulent pipe flows, where the velocity distribution can be described as follows:

$$
v(r)=v_{0} g(\varepsilon),
$$

where $g(\varepsilon)$ is a non-dimensional shape function ${ }^{[4]}$.

(4) becomes nonlinear in the case of open channel flows, as shown by experiment ${ }^{[9]}$ and numerical simulation ${ }^{[10]}$.

Figure 1 shows the case when the size of the magnets is much smaller than the width and height of the moving metal. We are interested in the $z$-component of the Lorentz force $F$ integrated over the volume of the conductor (which is equal to the force acting upon the magnets) as a function of the two geometry parameters, namely the non-dimensional filling parameter

$$
\xi=\frac{h}{\ell}
$$

and the nondimensional distance parameter

$$
\eta=\frac{w}{\ell} .
$$


We shall present our results both in dimensional and non-dimensional form. It has been shown by Thess et al. ${ }^{[4]}$ that the Lorentz force can be represented in the following form:

$$
F=C \sigma Q B_{0}^{2} \ell,
$$

where $Q$ is the volumetric flow rate (connected to the mean velocity as $Q=v_{0} h \ell$ ), $B_{0}$ is the magnetic field at a given reference position (the center of the bottom of the conductor in Fig. 2), and $\ell$ is a characteristic length scale of the problem. The dimensionless quantity $C$, which is the central focus of the present work, is either called calibration constant or calibration function. If the flow takes place in a fixed cross section, the quantity $C$ is indeed constant and its computation is straightforward ${ }^{[11]}$. If the cross section of the flow changes as in our present problem, $C$ depends on the geometry parameters of the problem. In our case, we have $C(\xi, \sigma)$, whose determination is the goal of our computation.

In what follows, we will use three kinds of velocity distributions. We start our computations with the solid body case when the velocity is uniform over the cross section of the conductor. We then perform simulations for an analytically given profile which represents the laminar solution of the Stokes problem with a free upper surface and no-slip boundary conditions at the side wall and at the bottom. We finally compare the results with a laminar Stokes flow where all four boundaries are solid walls at which the no-slip condition has to be satisfied.

The computations are limited to the kinematic case, which neglects the effect of the Lorentz forces acting on the flowing liquid. The influence of the magnetic field on the velocity distribution becomes significant when the magnetic interaction parameter $N=\sigma \ell B_{0}{ }^{2} /\left(\rho v_{0}\right)$ has values much greater than one ${ }^{[2]}$. This parameter has low values in our simulations: $N=4 \times 10^{-10}$ in the case of dipoles and $N=2.1$ in the case of finite size magnets, respectively. The magnetic effects on velocity profiles have been theoretically investigated by Hunt ${ }^{[12]}$ for laminar duct flows under the influence of uniform magnetic fields. The effects of non-uniform magnetic fields on turbulent and laminar flows have been numerically investigated in the previous work ${ }^{[8]}$.

\subsection{Solid conductor}

The numerical computations are performed with the finite element software COMSOL Multiphysics ${ }^{[13]}$. In the first step, numerical simulations are performed for a simple case of a solid conductor moving at constant velocity $v_{0}$. The magnetic field produced by two permanent magnets and the Lorentz force acting on the moving conductor are computed by using the magnetostatic application mode of the AC/DC COMSOL electromagnetic module. These electromagnetic computations have been validated in other simulation work ${ }^{[14]}$ by comparing numerical results with experimental data.

The magnetostatics application mode solves the coupled Ampère's law and the continuity equation written in terms of the electric potential $\phi$ and the magnetic vector potential $A$, i.e.,

$$
\begin{aligned}
& \nabla \times\left(\frac{1}{\mu} \nabla \times A-M\right)-\sigma v \times(\nabla \times A)+\sigma \nabla \phi=0, \\
& -\nabla \cdot(-\sigma v \times(\nabla \times A)+\sigma \nabla \phi)=0,
\end{aligned}
$$

where $M$ is the magnetization vector, $\boldsymbol{v}$ is the conductor velocity, and $\mu$ is the magnetic permeability. The Lorentz force acting on the conductor is computed by integrating the force density over the conductor volume.

The transversal cross section of the simulated configuration is presented in Fig. 2. The magnetization of dipoles is along the $x$-direction. Two finite size permanent magnets are also used in the simulations.

A surrounding air domain of dimensions few times larger than the characteristic dimension of the magnetic system have been considered in the simulation. The magnetic potential is set to zero at the air domain boundaries $A=0$. The electric insulation boundary condition $n \cdot J=0$ is imposed at the conductor walls. 


\subsection{Laminar flow}

For a laminar flow in a duct having a rectangular cross section of dimensions $\ell=2 a$ and $h=2 b$ in the $x y$-plane, the velocity distribution is given by ${ }^{[15]}$

$$
v(x, y)=\frac{3}{2} \frac{v_{0}}{1-6 \frac{b}{a} \sum_{n=1}^{\infty} \frac{1}{\alpha_{n}^{5}} \tanh \left(\alpha_{n} \frac{a}{b}\right)}\left(1-\frac{y^{2}}{b^{2}}+4 \sum_{n=1}^{\infty} \frac{(-1)^{n}}{\alpha_{n}^{3}} \frac{\cosh \left(\alpha_{n} \frac{x}{b}\right)}{\cosh \left(\alpha_{n} \frac{a}{b}\right)} \cos \left(\alpha_{n} \frac{y}{b}\right)\right),
$$

where $\alpha_{n}=\pi(2 n-1) / 2$. The mean velocity of the flow obtained by integrating the velocity distribution over the tube cross section is given by

$$
v_{0}=\frac{\chi+\rho g \sin \theta}{3 \tau} b^{2}\left(1-6 \frac{b}{a} \sum_{n=1}^{\infty} \frac{1}{\alpha_{n}{ }^{5}} \tanh \left(\alpha_{n} \frac{a}{b}\right)\right)
$$

where $\chi$ is the negative of the pressure gradient along the flow direction ( $z$-axis), $\rho$ is the density, $\tau$ is the dynamic viscosity, $g$ is the gravitational acceleration, and $\theta$ is the inclination angle of the tube. The velocity distribution for the laminar flow in an open channel of width $2 a$ and height $h$ is given by the same equations (11) and (12), where the half length $b$ is replaced by $h$. The vertical velocity profile on $y$-axis is half the complete parabolic profile in a laminar flow through a closed rectangular channel ${ }^{[15]}$.

The Navier-Stokes equations are solved for an incompressible Newtonian fluid by using the COMSOL Multiphysics. The simulation domain has the dimensions $2 a=0.01 \mathrm{~m}, 2 b=0.005 \mathrm{~m}$ and the length $L=1 \mathrm{~m}$. In order to have a fully developed flow, the channel length must be greater than the entrance length $L_{\mathrm{e}}$, which is given by

$$
L_{\mathrm{e}} \approx 0.06 \ell R e
$$

where $\ell=2 a$ is the channel width. The Reynolds number is defined as

$$
R e=\frac{4 \rho R_{\mathrm{h}} v_{0}}{\tau},
$$

where the hydraulic radius $R_{\mathrm{h}}$ is the ratio between the cross-sectional area and the wetted perimeter.

At the inlet boundary, the input velocity is set to $v_{0}$, and at the outlet boundary the pressure is $p=0$. The no-slip boundary condition $(v=0)$ is imposed along the channel walls and no shear stress condition is used at the free surface.

The numerically computed velocity profiles on the vertical $y$-axis compared with the analytical predictions given by (11) for closed and open channel flow, are shown in Fig. 3. The velocity profiles are computed in the absence of the magnetic field. The small differences between numerical and analytical results are due to the graphical plot of (11). This equation contains an infinite sum which cannot be plotted with our graphics software. The agreement is improved when more terms are added in our function plot. Therefore, both the numerical computations and (11) can be used to predict the velocity distribution in laminar closed and open channel flow. The flow simulations for the unidirectional profile have been carried out for the purpose of the verification of the code. 

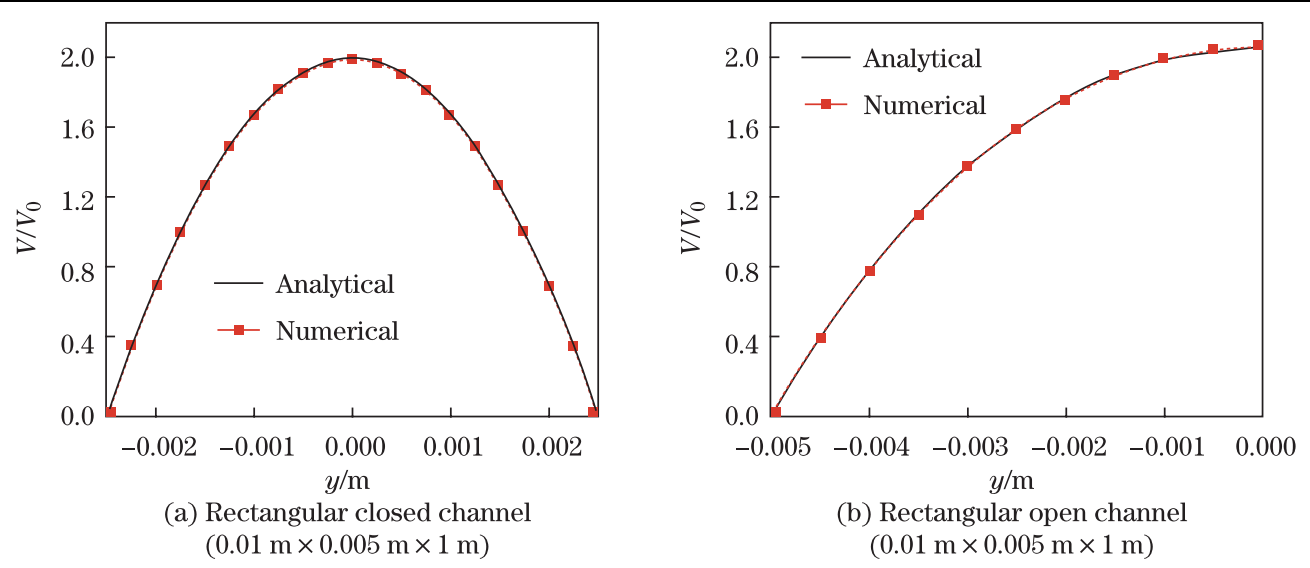

Fig. 3 Comparison of numerical and analytical velocity profiles along $y$-axis for laminar flow

\section{Numerical results for magnetic point dipoles}

Two small dipoles of dimensions $0.0004 \mathrm{~m} \times 0.0002 \mathrm{~m} \times 0.0002 \mathrm{~m}$ are used in our simulations. In this way, the magnetic field inhomogeneity due to the shape and magnets dimensions are minimal. The magnetization of dipoles in the $x$-direction is $M_{x}=10^{6} \mathrm{~A} / \mathrm{m}$. The conductor has the width $\ell=0.01 \mathrm{~m}$ and the length $L=0.1 \mathrm{~m}$. The distance between the conductor and dipoles $(w)$ and the conductor height $(h)$ are varied over the ranges: $w=0.001 \mathrm{~m}-0.01 \mathrm{~m}$ $(\eta=0.1-1)$ and $h=0.001 \mathrm{~m}-0.02 \mathrm{~m}(\xi=0.1-1)$. The Lorentz forces are computed at constant velocity $v_{0}=1 \mathrm{~m} / \mathrm{s}$, then the dimensionless calibration coefficient is computed by using (8), where the electrical conductivity of the solid body is $\sigma=10^{6} \Omega^{-1} \cdot \mathrm{m}^{-1}$, the volumetric rate is $Q=v_{0} \ell h$, and $B_{0}$ is the $x$ component of the magnetic induction in a point located at the bottom surface of the conductor (see Fig. 2).

The results obtained by varying the conductor height $h$ (dimensionless quantity $\xi$ ) and the distance $w$ of the magnetic dipoles from the conductor (dimensionless quantity $\eta$ ) are plotted in Fig. 4. The calibration coefficient first increases to a maximum value, then decreases when the conductor height is augmented beyond a critical value. The existence of a maximum $C$ versus $\xi$ indicates that for this particular filling level a Lorentz force flow meter has its highest sensitivity. This observation implies that the distance between the magnets of the flow meter sketched in Fig. 1 should be chosen in such a way that the most probable filling level corresponds to the filling level at which the sensitivity is maximum.

The existence of a maximum of $C(\xi)$ is a consequence of the fact that $C=0$ both for $\xi=0$ and for $\xi \rightarrow \infty$. The first fact, i.e., $C(0)=0$, follows from the observation that the dominant source of the eddy currents is the electromotive force proportional to the $x$-component of the magnetic field and the $z$-component of the velocity. As $\xi \rightarrow 0$, these currents become confined and tend to zero as $h^{2}$. The decay of $C$ for $\xi \rightarrow \infty$ is due to the fact that for increasing fluid height an increasing part of the conductor is located in a region where the magnetic field is very weak. Since the calibration constant is a weighted average of contributions from all $h$, the contributions from the distant parts of the conductor lead to the asymptotic decay of $C$. The value of $\xi$ corresponding to a maximum of $C$ increases with increasing distance between the magnets and the conductor (see Fig. 4).

Figure 4 permits a systematic evaluation of the influence of the velocity profile on the calibration coefficient. Figure 4(a) shows that the calibration coefficient of a moving solid body (blue line) is roughly twice as high as those of the laminar flow of a fluid. Figures 4(b)$4(\mathrm{~d})$ indicate that this difference becomes smaller when the distance between the magnet and 
conductor increases. This is due to the fact that for small $\eta$ the magnets "feel" the velocity gradient near the wall stronger than for high $\eta$. These features indicate that a dry calibration procedure does not in general provide calibration coefficients that are close to those in a laminar flow. However, it could also be noted that the ratio between the solid and liquid curves in Figure 4 are roughly independent of $\xi$. Hence, it could be possible to recalculate calibration coefficients for liquid metals from dry calibration experiments. Another aspect in favor of dry calibration is that most flows in metallurgy are turbulent and their velocity profiles are in between those of a solid body and a laminar flow. Hence, the difference between dry calibration and liquid calibration for turbulent flows is smaller than the curves in Fig. 4.

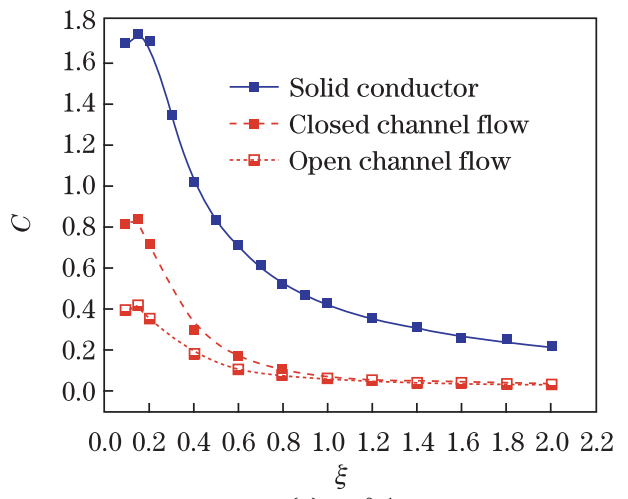

(a) $\eta=0.1$

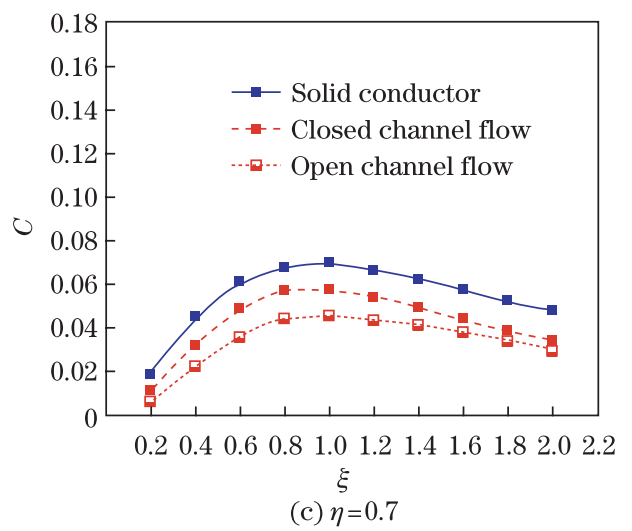

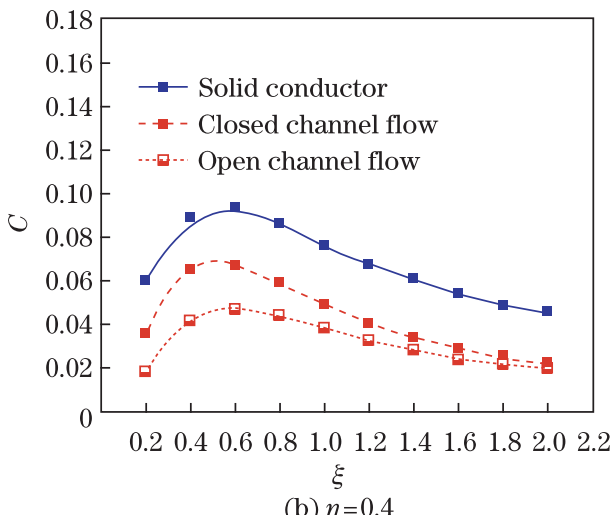

(b) $\eta=0.4$

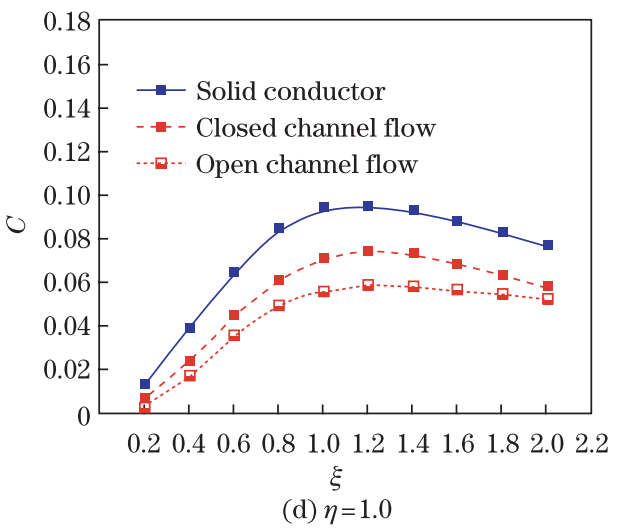

Fig. 4 Dimensionless calibration coefficient $C$ versus dimensionless height $\xi$ for solid conductor, closed channel flow, and open channel flow at different distances between dipoles and conductor $(\eta=w / \ell)$

\section{Numerical computations for finite size magnets}

The influence of the magnet dimensions on the calibration function is analyzed by increasing the magnets size. The simulations are performed for the configuration shown in Fig. 5(a), with two cubic magnets of $0.01 \mathrm{~m}$ length size, distanced by $d=0.03 \mathrm{~m}$, and having the magnetization $M_{x}=2 \cdot 10^{6} \mathrm{~A} / \mathrm{m}$. The channel has the dimensions $0.01 \mathrm{~m} \times 0.01 \mathrm{~m} \times 0.7 \mathrm{~m}$ in the $x-, y-$, and $z$-directions. The centers of the magnetic system, the channel (in the $x y$-plane), and the coordinate system coincide. The magnetic field distribution in the channel is non-uniform in the $x$-, $y$-, and $z$-directions (see Fig. 5(b)).

The calibration coefficients are computed in the case of a solid conductor and the laminar flow in closed and open channels. The liquid height is varied over the range $h=0.002 \mathrm{~m}-0.02 \mathrm{~m}$ 

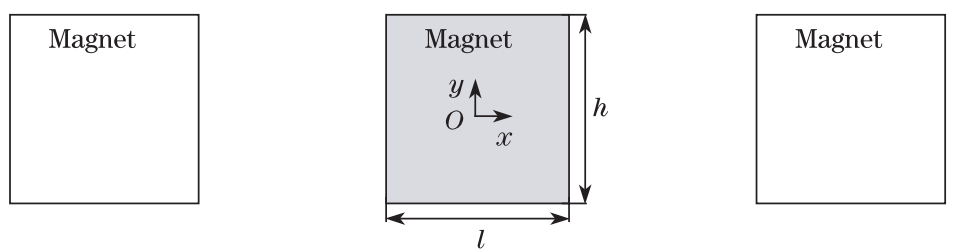

(a) Transversal cross section of simulated domain with two cubic magnets
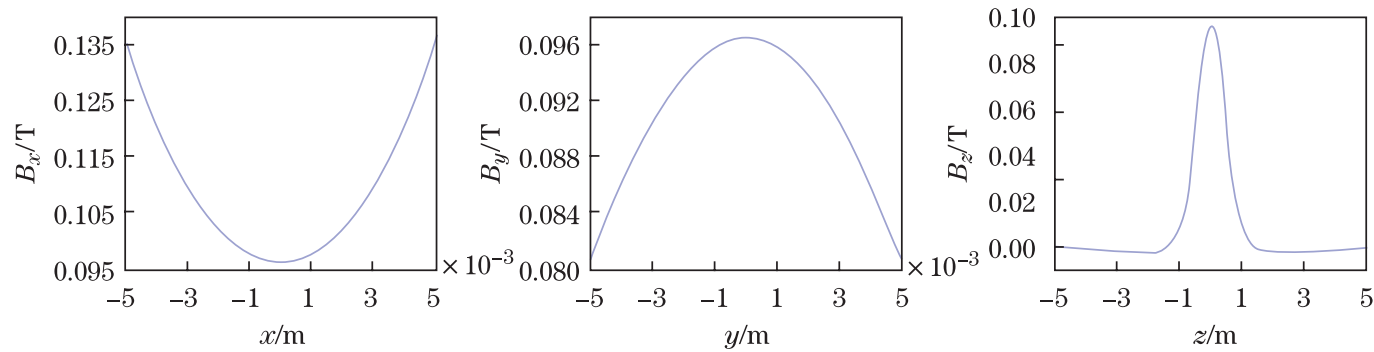

(b) Magnetic induction $B_{x}$ on $x$-, $y$-, and $z$-axes

Fig. 5 Transversal cross section of simulated domain with two cubic magnets and magnetic induction $B_{x}$ on $x$-, $y$-, and $z$-axes

(see Fig. 6). The input velocity in the computations is the mean velocity of the flow $v_{0}$, which in laminar open channel flow is related to the liquid height $h$ by (12). The computations performed by accounting for the laminar flow effects show significant differences in the resulting Lorentz forces and calibration coefficients in comparison with the solid body case. The maximum error about the estimation of the calibration function by using the simplified solid body computations, is $\delta C \approx 38 \%$. However, these errors are much smaller as compared with computations performed for magnetic dipoles (see Fig. 4).

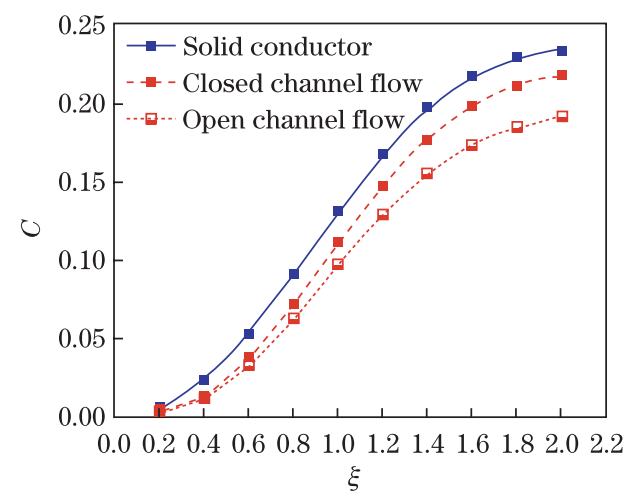

Fig. 6 Dimensionless calibration coefficient $C$ versus dimensionless height $\xi$ for solid conductor, closed channel laminar flow, and channel laminar flow in case of finite size magnets

The Lorentz forces and the calibration coefficients carried out from computations including velocity effects are smaller than the same quantities carried out from solid body computations. This can be explained by different patterns of induced eddy currents, which depend on both the velocity and magnetic field distribution in the liquid metal. These effects are analyzed by plotting the velocity profiles, eddy currents and Lorentz forces computed for a solid conductor (see Fig. 7), and a duct laminar flow (see Fig. 8). The simulations are performed for the 
configuration shown in Fig. 5(a). The magnets are located at $z=0$ along the flow direction ( $z$-axis). The mean velocity of the flow is $v_{0}=0.02 \mathrm{~m} / \mathrm{s}$, which corresponds to a Reynolds number $R e=500\left(\rho=2370 \mathrm{~kg} / \mathrm{m}^{3}, \tau=0.001 \mathrm{~N} \cdot \mathrm{s} / \mathrm{m}^{2}\right)$.

In the case of a solid conductor, the velocity is constant in the $x$-, $y$-, and $z$-axes (see Fig. 7(a)). The induced eddy currents are plotted in Fig. 7(b). The color maps show eddy currents distribution in the transversal $x y$-plane and the longitudinal $y z$-plane. The magnets are shown in the $x y$-section. The component of eddy currents parallel to the $y$-axis contributes to the measureable $F_{z}$ Lorentz force for a LFF device. The negative $j_{y}$ currents induce negative (damping) $F_{z}$ forces, while positive $j_{y}$ currents induce positive (accelerating) $F_{z}$ forces. Eddy currents are closed in the longitudinal $y z$-plane, due to the magnetic field non-homogeneity. The component $j_{y}$ is negative in the region with high magnetic field and positive in the region where the magnetic field vanishes. The $x$, and $y$-axes plots show only negative values of $j_{y}$,
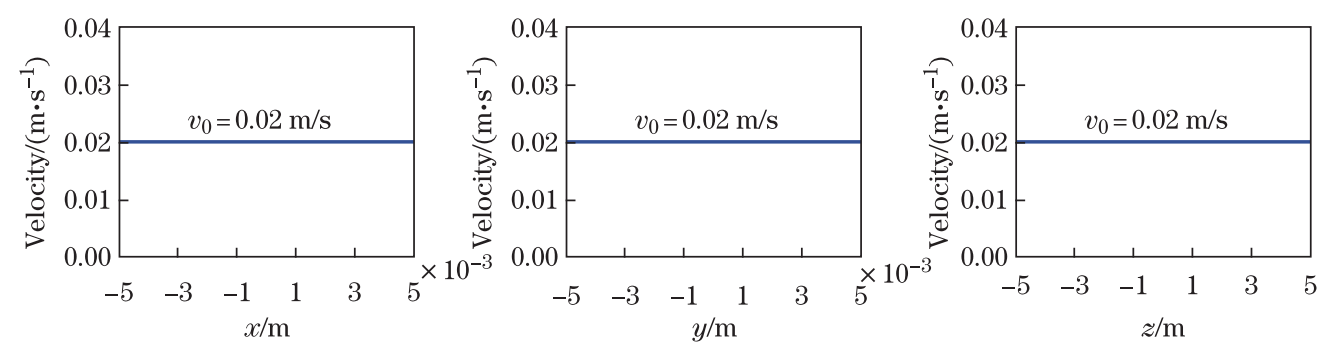

(a) Velocity profile

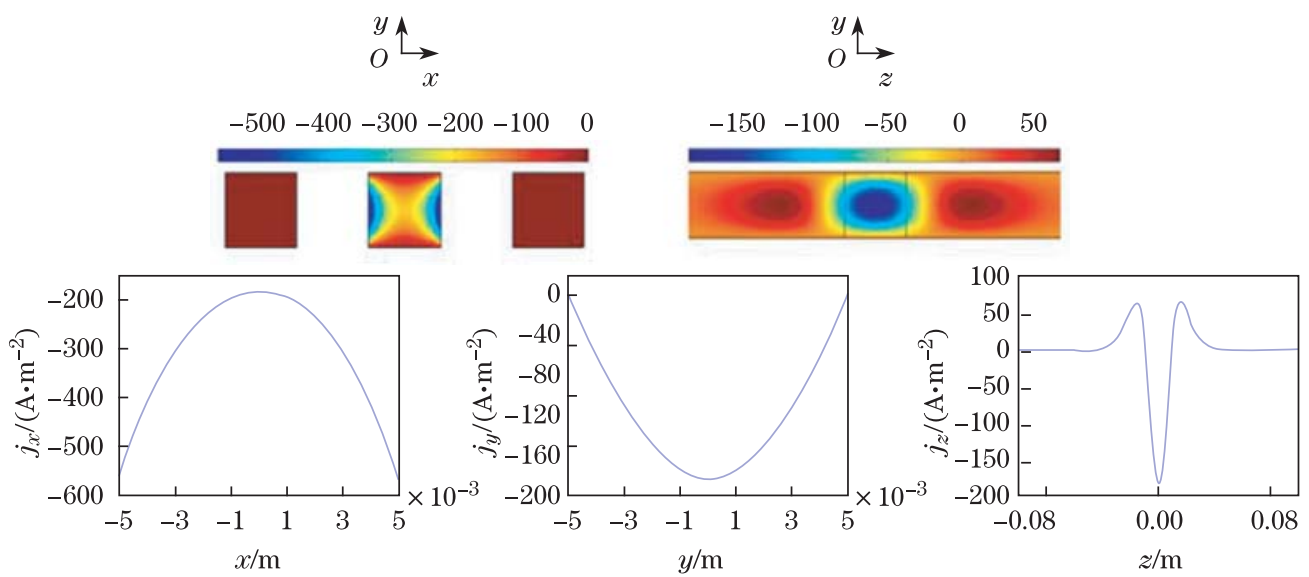

(b) Eddy current density $j_{y}$ (color maps in $x y$ - and $y z$-sections)
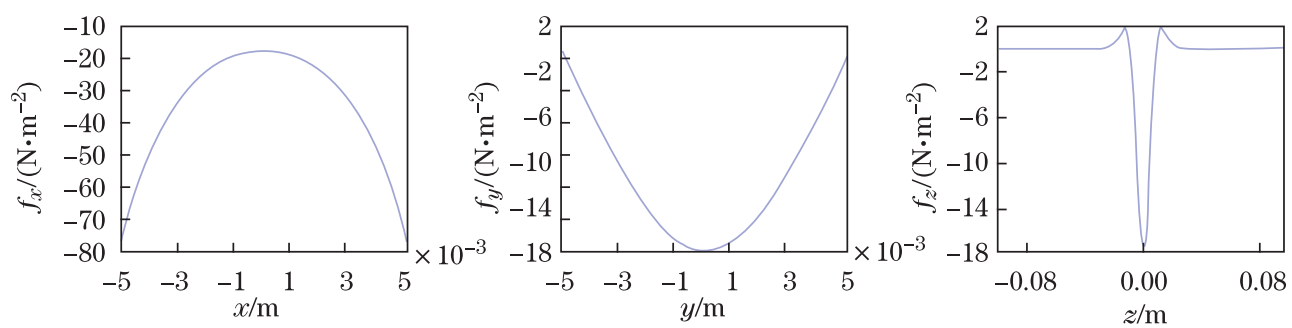

(c) Lorentz force density $f_{z}$

Fig. 7 Numerical plots on $x-, y$-, and $z$-axes for solid conductor moving at $v_{0}=0.02 \mathrm{~m} / \mathrm{s}$ (velocity profile, eddy current density $j_{y}$ (color maps in $x y$ - and $y z$-sections), and Lorentz force density $f_{z}$ ) 
which means that there is no closed path of currents in the transversal $x y$-cross section. The Lorentz force density plotted in Fig. 7(c) shows a predominant negative $F_{z}$ component.

The velocity profiles, eddy currents, and Lorentz forces carried out from laminar flow computations are plotted in Fig. 8 . The velocity profiles have a parabolic shape in the $x$ - and $y$-axes (see Fig. 8(a)). The plot along the flow direction ( $z$-axis) shows an increasing velocity over the entrance length $\left(L_{\mathrm{e}} \approx 0.3 \mathrm{~m}\right)$. Velocity boundary layers appearing near the walls create a supplementary difference of the electrical potential in the transversal plane. Therefore, eddy currents can be closed in the transversal $x y$-cross section, as shown in Fig. 8(b). The $x$ plot shows negative currents in the core region of the channel, and positive currents in the wall boundaries of about $0.0015 \mathrm{~m}$ width. The $z$ plot shows also the closure of eddy currents in the longitudinal $y z$-plane, as for a solid conductor. Eddy currents which turn in the transversal cross section create positive $F_{z}$ forces, as shown in Fig. 8(c). Finally, the integrated (negative) $F_{z}$ force is significantly reduced for a laminar flow $\left(F=-1.54 \times 10^{-5} \mathrm{~N}\right)$ as compared with the solid body case $\left(F_{\mathrm{S}}=-1.612 \times 10^{-5} \mathrm{~N}\right)$. The relative error about solid body computations of
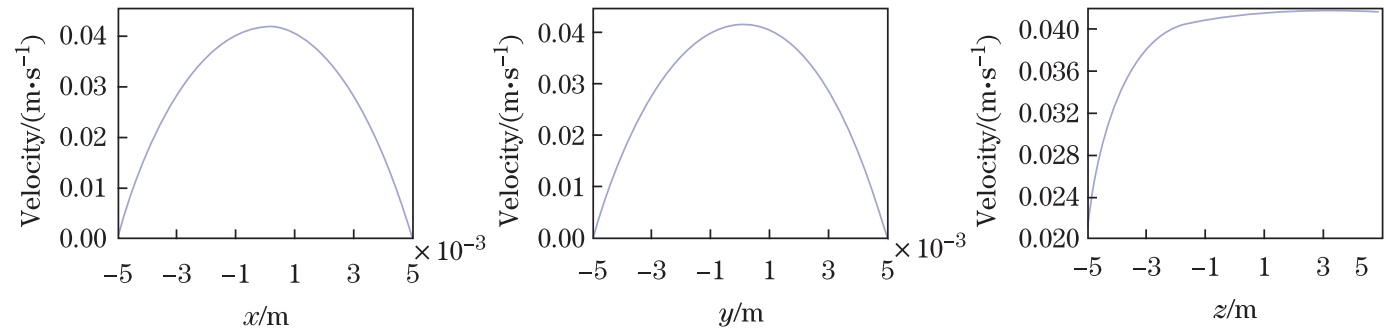

(a) Velocity profile
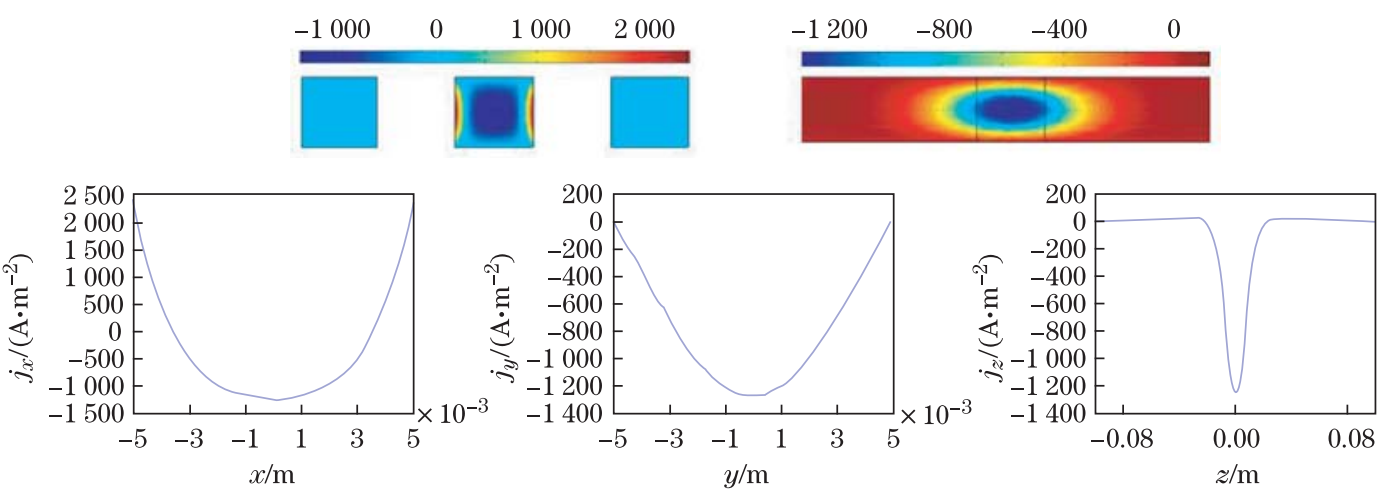

(b) Eddy currents density $j_{y}$ (color maps in $x y$ - and $y z$-sections)
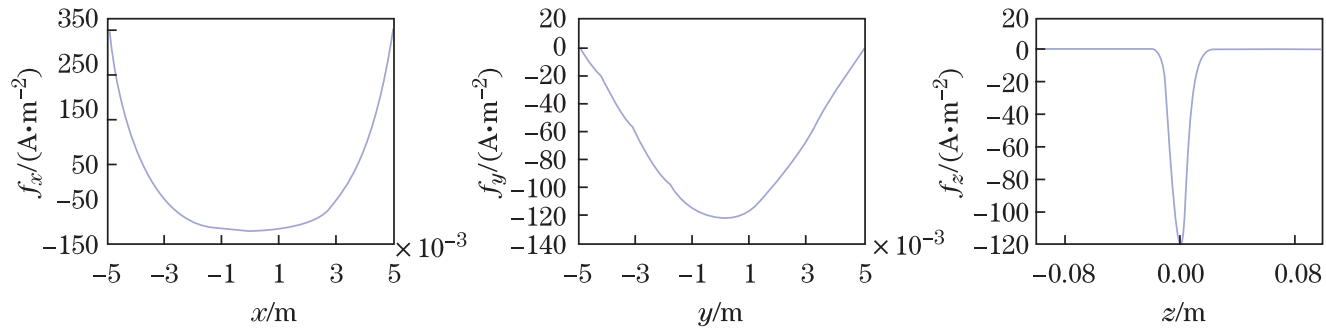

(c) Lorentz force density $f_{z}$

Fig. 8 Numerical plots on $x$-, $y$-, and $z$-axes for laminar flow without magnetic field effects (velocity profile, eddy current density $j_{y}$ (color maps in $x y$ - and $y z$-sections), and Lorentz force density $\left.f_{z}\right)$ 
the Lorentz forces given by $\delta F=\left(F_{\mathrm{S}}-F\right) / F_{\mathrm{S}}$ is $\delta F=4.5 \%$ for the simulated case. Therefore, we conclude that the velocity effects would be accounted for in the LFF numerical calibration procedure.

\section{Conclusions}

The influence of the laminar velocity profile on the calibration function of a Lorentz force flowmeter is numerically investigated by using COMSOL Multiphysics. Computations performed for laminar flows in closed and open rectangular channels are compared with a simple case of a conductor moving at the mean velocity of the flow.

The laminar flow computations are first validated by comparing the numerical velocity profiles with theoretical predictions. Then, velocity effects are accounted for in the numerical computations of the Lorentz forces. It is found that the laminar velocity profiles has a significant effect on the resulting Lorentz forces. These effects are explained by different patterns and densities of the induced eddy currents in laminar flows and solid body computations. Numerical computations of the calibration function show significant errors if the simplified solid body computations are used in the calibration procedure. These errors increase up to $\delta C=75 \%$ in the case of dipoles and $\delta C=38 \%$ in the case of finite size magnets. Finally, one can conclude that the calibration function of a Lorentz force flowmeter is significantly influenced by the velocity distribution in laminar closed and open channel flow. Therefore, laminar flow computations should be included in the LFF calibration procedure.

Acknowledgements The authors are grateful to the German Research Foundation (Deutsche Forschungsgemeinschaft) for supporting the work in frame of the Research Training Group (Graduiertenkolleg) "Lorentz force velocimetry and Lorentz force eddy current testing" at Ilmenau University of Technology. The authors also acknowledge helpful discussion with Prof. S. MOLOKOV of Coventry University.

\section{References}

[1] Davidson, P. A. An Introduction to Magnetohydrodynamics, Cambridge University Press, Cambridge (2001)

[2] Shercliff, J. The Theory of Electromagnetic Flow Measurements, Cambridge University Press, Cambridge (1962)

[3] Thess, A., Votyakov, E. V., and Kolesnikov, Y. Lorentz force velocimetry. Physical Review Letters, 96, $164501(2006)$

[4] Thess, A., Votyakov, E. V., Knaepen, B., and Zikanov, O. Theory of the Lorentz force flowmeter. New Journal of Physics, 9, 299 (2007)

[5] Kolesnikov, Y., Karcher, C., and Thess, A. Lorentz force flowmeter for aluminium: laboratory experiments and plant tests. Metallurgical and Materials Transactions B, 42(3), 441-450 (2011)

[6] Priede, J., Buchenau, D., and Gerbeth, G. Single-magnet rotary flowmeter for liquid metals. Journal of Applied Physics, 110, 034512 (2011)

[7] Minchenya, V., Karcher, C., Kolesnikov, Y., and Thess, A. Dry calibration of the Lorentz force flowmeter. Magnetohydrodynamics, 45(4), 569-578 (2009)

[8] Stelian, C. Analysis of turbulent flow in closed and open channels with application in electromagnetic velocimetry. Magnetohydrodynamics, 48(4), 503-515 (2012)

[9] Minchenya, V., Karcher, C., Kolesnikov, Y., and Thess, A. Calibration of the Lorentz force flowmeter. Flow Measurement and Instrumentation, 22, 242-247 (2012)

[10] Stelian, C. Calibration of a Lorentz force flowmeter by using numerical modeling. Flow Measurement and Instrumentation, 33, 36-44 (2013)

[11] Wang, X., Kolesnikov, Y., and Thess, A. Numerical calibration of a Lorentz force flowmeter. Measurement Science and Technology, 23, 045005 (2012) 
[12] Hunt, J. C. R. Magnetohydrodynamic flow in rectangular ducts. Journal of Fluid Mechanics, 3, 37-62 (1971)

[13] COMSOL, A. B. The COMSOL Multiphysics Reference Guide, COMSOL Office, Sweden (2008)

[14] Stelian, C., Alferenok, A., Lüdtke, U., Kolesnikov, Y., and Thess, A. Optimization of a Lorentz force flowmeter by using the numerical modeling. Magnetohydrodynamics, 47(3), 273-282 (2011)

[15] Pozrikidis, C. Fluid Dynamics: Theory, Computation and Numerical Simulation, Springer, New York (2009) 